\title{
LAS ORGANIZACIONES SENSIBLES, COHERENTES Y LUCIDAS (OSCL): SU INTEGRIDAD Y ESTRUCTURA COGNITIVA - VALORATIVA
}

\section{SENSITIVE , COHERENT AND LUCID ORGANIZATIONS ( OSCL ) : INTEGRITY AND COGNITIVE STRUCTURE - EVALUATIVE}

\section{RESUMEN}

Una empresa que adopta la gestión del conocimiento en su modelo OSCL garantiza que gana en identidad y en arquitectura empresarial mediante la conducción del flujo organizacional en estados de perturbación o turbulencia, logrando arquetipos de labor disciplinada en estados de movilidad estática o dinámica y orden simple o complejo desde los cuales se valora direcciona y conduce la empresa hacia la cultura del éxito desde el desarrollo de los sujetos hacia y en el desarrollo empresarial impactando y estimulando el desarrollo social.

Palabras clave: Sostenibilidad ambiental, agilidad, competitividad, gestión del conocimiento, valoración, riesgo, incertidumbre, colaboración, innovación, normas, identidad.

\begin{abstract}
A company that adopts knowledge management model ensures that wins OSCL identity and enterprise architecture by conducting organizational flow in states of disturbance or turbulence , making archetypes of disciplined work in states of static or dynamic mobility and simple command or complex values from which directs and leads the company towards the culture of success from the development of the subject to and impact on business development and stimulating social development.
\end{abstract}

Keywords: Environmental sustainability , flexibility , competitiveness , knowledge management, valuation, risk, uncertainty, collaboration, innovation, standards, identity.

\section{INTRODUCCIÓN}

La expresión Organizaciones Sensibles, Coherentes y Lucidas (O.S.C.L.) describe una entidad de CARÁCTER INGENIOSO que aborda sus dependencias de una manera fundamentalmente diferente de las actuales empresas del orden productivo, donde emergen la solidez corporativa (Penetración, estabilidad, credibilidad, tiempo y flexibilidad) y la

\section{Liliana Astrid Bejarano Garzón:}

Ingeniero de Sistemas - Universidad Piloto de Colombia - País. Especialista en Docencia Universitaria - Universidad del Rosario - País. Colombia Docente Investigadora - Universidad Distrital - Colombia -

Tipo: Artículo Reflexión

Fecha de Recepción: 04-Abr-2014

Fecha de Aceptación: 24-Jun-2015 
firmeza empresarial (costos, calidad, servicio, credibilidad, tiempo y flexibilidad) como enriquecedores valiosos del discurrir en permanencia y vigencia.

El esquema de las O.S.C.L. es un mapa de caminos que describe cómo logran las compañías exitosas obtener beneficios a largo plazo en las dimensiones de la competencia. Los cuatro requisitos estructurales son mejoramiento continuo, investigaciones y desarrollo, adopción de tecnología avanzada e integración de personas, sus valores y sistemas, habilitan a una empresa para competir de manera simultánea en todas estas dimensiones. La estrategia de la empresa coordina sus esfuerzos y la conduce hacia sus metas. [29]

Si una empresa quiere convertirse en una organización sensible, coherente y lúcida (OSCL) debe de:

(a) Basar su COLUMNA VERTEBRAL de IDENTIDAD SISTÉMICA (CVIS) en la interacción altamente dinámica y compleja de sus tres ejes flexibles de identidad estructural - funcionalista a saber: 1 . Conjugacional 2. Cognitiva y 3. Axiológica.

(b) Expresar su PENSAMIENTO DE ARQUITECTURA EMPRESARIAL (PAE) en términos de la interacción compleja de sus componentes: 1 . Direccional, 2. Accional y 3. Actitudinal.

Debe ser contextualizado y guiado por los conceptos de:

- $\quad$ Calidad

- $\quad$ Núcleo Solicitante Expectante (NSE)

- Fuentes de Mantenimiento y renovación (FMR)

- $\quad$ Normatividad

El PAE es la fuerza motriz de la matriz de Soporte Estructural y Funcional (SEF), quien a su turno posibilita a la empresa alcanzar su crecimiento y desarrollo al realizar sincrónicamente todas las actividades de los vectores de la matriz de expansión, con lo cual la firmeza empresarial y la solidez corporativa emergen como enriquecedores valiosos del discurrir en permanencia y vigencia constituyéndose así en el paradigma de cultura empresarial exitosa. Este modelo para el diseño y conformación de las O.S.C.L. es de la autora del artículo, el cual busca estructurar un complejo que garantice la calidad del proceso; que soporte el PAE de la empresa y lleve a la firma a alcanzar sus metas con éxito.

\section{PRESENTACIÓN DEL DOCUMENTO}

Una empresa como OSCL es de alta complejidad y esbelta presencia holística puesto que su concepción se da para discurrir en ambientes de alta turbulencia, variabilidad y variedad, lo cual significa un gran espectro de contingencias e incertidumbres por superar [1][2][3]. La propuesta es alcanzar el éxito empresarial mediante la gestación y maduración de una cultura organizacional constructiva, basada en el desarrollo personal del agente activo como sujeto y el desarrollo integro de la compañía como sociedad productiva sustancial del sistema económico mundial, esto lleva indiscutiblemente a precisar los soportes clásicos de CERTIFICACIÓN DE CALIDAD Y RIGUROSIDAD en el cumplimiento de la normatividad, lo cual establecería la legalidad INSTITUCIONAL y complementariamente desde la ECOLOGÍA PROFUNDA embebida en la FIRMEZA EMPRESARIAL Y LA SOLIDEZ CORPORATIVA el aseguramiento de la responsabilidad social empresarial a la vez que la aceptación de la organización como patrimonio e institución empresarial por parte de la sociedad.

El escenario conceptual descriptivo de una organización sensible, coherente y lúcida (O.S.C.L.) está compuesto por: 1.Satisfacción del núcleo solicitante expectante. 2. Competir simultáneamente con los vectores de la matriz de competencia. Permite a una empresa satisfacer al núcleo solicitante / expectante. 3. Matriz de competencia (costos, credibilidad, calidad, flexibilidad, tiempo, servicio, penetración, estabilidad). 4. Los soportes estructurales posibilitan a la empresa competir simultáneamente con todos los vectores de la matriz competencia. 5. Matriz de soporte estructural (vectores y dimensiones). 6. La estrategia es la fuerza motriz de la matriz de soporte estructural. 7 . Estrategia orientada al núcleo solicitante / expectante. 


\section{DISCUSIÓN}

Una OSCL está diseñada con esbelta presencia holística que bien se podría traducir como un sistema abierto cuyo desempeño relaciona no solo los aspectos vertebrales del proceso de transformación con su medio interno, sino que, involucra de manera activa y significante su relación con el medio ambiente externo: 1 . Inmediato ó Proximal (I/P) sino el 2. Mediato o medial (M/M) y 3. El Tardío o Lejano (T/L), en la mirada tripartita Espacio, Tiempo, Corporeidad. (E.T.C), como se ilustra en la Figura 1.

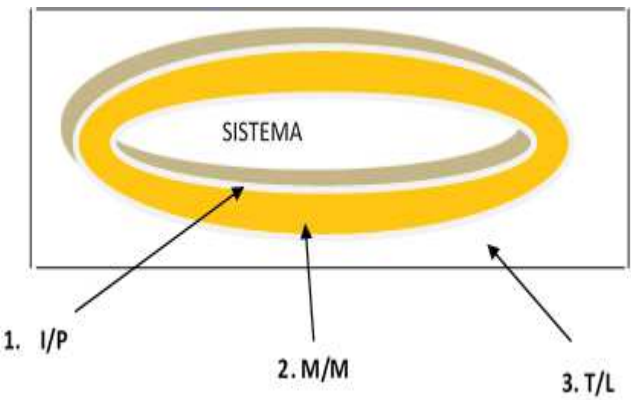

Figura 1. Aspectos vertebrales y su relación con el medio ambiente. (Fuente: Propia)

$$
\begin{aligned}
& \varphi_{d}=\phi_{\text {int }} \rho^{*} \phi_{\text {ext }} \rho \\
& \varphi_{d}=\rho\left(\phi_{\text {int }} \phi_{\text {ext }}\right)
\end{aligned}
$$

$$
\begin{aligned}
& \text { Donde: } \\
& \varphi_{d}=\text { Vector flujo del desempeño } \\
& \phi_{\text {int }}=\text { Vector flujo del desempeño en el medio ambiente interno. } \\
& \phi_{\text {ext }}=\text { Vector flujo del desempeño con el medio ambiente externo. } \\
& \rho=\text { Función de configuración compleja. }
\end{aligned}
$$

El vector de flujo de desempeño organizacional $(\varphi \mathrm{d})$ puede ser visto por la relación entre los flujos de desempeño interno (фint) y flujo de desempeño externo ( $\phi$ ext), mediante la función de conFiguración compleja ( $\rho$ ), en el domi- nio tripartita (E.T.C).

Desde el punto de vista OSCL, la empresa o el negocio que ella encarna será fluida o laminar hasta tanto la TURBULENCIA $(\tau)$ no se presente o sea manejada con criterios trasdisciplinares. Tanto la variedad (Ve) como la variabilidad (Vb) y su interacción son factores vectoriales que dirigen la TURBULENCIA según prime el producto punto, el cruz extendido entre ellas, manifestándose la $\tau$ en la caída del producto o servicio $(\Lambda S)$, frenetiques financiera (Ff), cambios drásticos del mercado $(\mathrm{dm})$ o en los fractales comerciales del paradigma económico de referencia.[4]

Los criterios y conceptos aquí utilizados vienen desde la mirada holística de la Teoría General de Sistemas (TGS) tangibilizada en primera instancia por el enfoque sistémico que se le da al CAOS en la versión de complejidad llamándole adaptación desde la simplicidad de la macro referencia evolución. Ahora bien siguiendo en la misma línea pero siendo técnicamente ingenieriles la OSCL manifiesta tanto resiliencia organizacional como la autopoiesis empresarial como propiedades fundantes para manejar no solo las emergencias y las contingencias,[5] desde las interacciones de acomodación o adaptación sino desde el incremento variacional (producto punto) el incremento de variedad (producto cruz) incremento fuerte de variabilidad (producto cruz extendido). Logrando con este enfoque organizacional desarrollo y crecimiento empresarial holístico.

Debe quedar claro que el flujo organizacional está compuesto por los vectores característicos de la función del negocio[6][7][8] y este libre flujo mantiene íntima relación con los flujos de desempeño a la vez que con el subsistema VIDAL (medición y recompensa) y concomitantemente con el subsistema de retribución e incentivos (REI) esta composición en red de redes de la organización bajo el modelo de efectividad[9][10][11] SORI (Solidez, Operaciones, Resultados, Intenciones) proporciona estabilidad y crecimiento organizacional una vez que tanto en pre flujo turbulento como en el mismo la autopoiesis empresarial permite generar micro autocontroles que en interacción con otros 
del mismo nivel o conFiguraciones auto controladas [12][13][14] de diferentes niveles aseguran que las Operaciones Misionales Vertebrales (OMV) permanezcan o migren suavemente a las Nuevas Operaciones Misionales Vertebrales (OMV),[15][16][17] lográndose con ello la misión o actuación empresarial bajo turbulencia (emergencia, contingencia) que permite obtener resultados del Negocio en el equilibrio dinámico a homeostasis empresarial [18][19] [20] del cual se derivará la experiencia empresarial que se expresará en la misión contingente y en la proyección tanto de la nueva misión organizacional como de la visión institucional derivada de la sensibilidad a los cambios en el medio ambiente interno[21][22] [23] como en el externo y restaurada por la función resiliencia del subsistema prospector con sus vectores de descripción y acción de las futuribles del sistema.

Ante el espectro de la perturbación o en la alta turbulencia [24][30] las funciones de reordenamiento inicial y las de mayor resiliencia reclaman el cultivo de:

1. Conocimiento de la identidad empresarial.

2. Prospectiva de la marca institucional.

3. Hidalguía y proposición emocional en todos los frentes.

4. Aprender de la perturbación o turbulencia (Experiencia).

5. Reconocimiento del momentum crítico (sensibilidad)

6. Pedir y proporcionar ayuda efectiva (Lucidez)

7. Desarrollar la conducta o comportamiento vital positivo y efectivo

Esta cultura de renovación [25] [26] [27] [30] ante la perturbación o turbulencia es una seudo gestación organizacional que provee flexibilidad y oportunidad para desarrollar el sistema vital de sustentabilidad.

La OSCL es sensible y se nutre del cambio o transformación, que puede o no seguir un curso lógico según el tipo de perturbación a enfrentar que puede ser bajo distintas condiciones: Continuas, inevitables, graduales, abruptas, lo- cales, globales, requeridas o no, fomentadas o inesperadas. Así es un hecho que aun la menor de las perturbaciones originará un cambio, esta transformación originada en una resiliencia menor o mayor incrementará el POTENCIAL DE TRANSFORMACION, es decir se aumentan las posibilidades de pasar a un estado o conFiguración organizacional diferente que propugne por una tendencia al EQUILIBRIO DINÂMICO ó ESTÁTICO donde el flujo organizacional tenga comportamiento laminar. [32][33]

La caracterización de una OSCL según se mire el orden (CAOS) y la movilidad (EQUILIBRIO) permite tomar un referente o dominio particular para tomar decisiones desde el deber hacer al deber ser buscando la efectividad en el deber PERMANECER. [34][35][36]

A modo de ejemplo si el CAOS, se ve desde el orden dicotomizando se tendrían dos estados SIMPLE y COMPLEJO de igual forma el EQUILIBRIO desde el CAMBIO o movilidad los dos estados serían ESTÁTICO y DINÁMICO cruzando estas dos dimensiones movilidad - orden. Se obtienen cuatro estados característicos de organización:

1. Delineación fabril

2. Diseño empresarial

3. Composición corporativa

4. Arquitectura institucional

A estos arquetipos le corresponderían identificaciones de acciones misionales y/o visionales según el riesgo y la incertidumbre concomitantes, que a su turno se asociarían a acciones de investigación y desarrollo concretadas en innovaciones y/o creación en productos y/o procesos según se puede reflexionar en los infoesquemas.

1. Arquetipos organizacionales. Ver Figura 2

2. ConFiguraciones identificatorias. Ver Figura. 3

3. Patrones de acción vital organizacional. Ver Figura 4. 


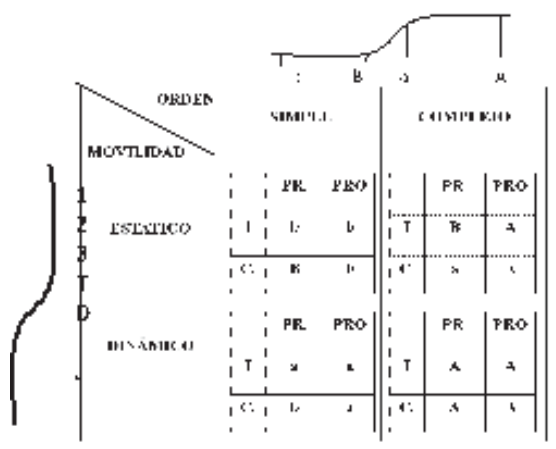

PR $=$ Producto, $\mathrm{PRO} .=$ Proceso, $\mathrm{I}=$ Innovación, C. $=$ Creatividad, $\mathrm{a}=$ Nivel mas bajo del alto, $\mathrm{A}$ $=$ Nivel más alto del alto. $\mathrm{b}=$ Nivel mas bajo del bajo y $\mathrm{B}=$ Nivel mas alto del bajo. 1 = Equilibrio Indiferente, 2 = Equilibrio Estable, $3=$ Equilibrio Inestable, $\mathrm{T}=\mathrm{AlTo}, \mathrm{D}=\mathrm{MeDio}, \mathrm{J}=\mathrm{BaJo}$.

Figura 2. Arquetipos Organizacionales. (Fuente: Propia)

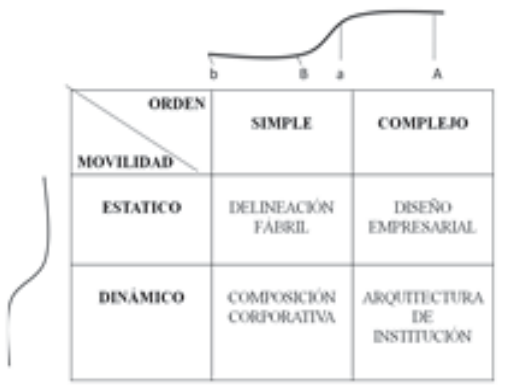

Figura 3. ConFiguraciones identificatorias (Fuente: Propia)

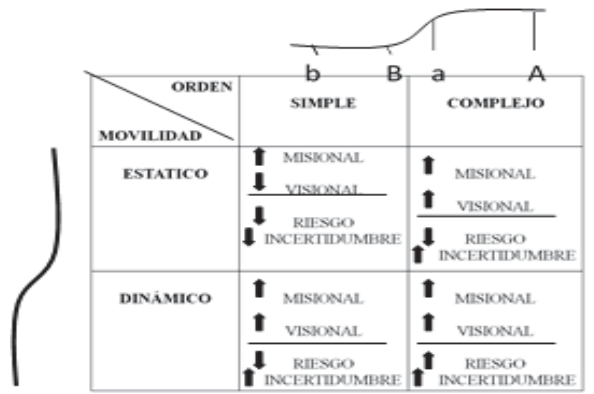

Figura 4. Patrones de acción vital organizacional (Fuente: Propia)
El sostén vital en lucidez está marcado por un cultivo transversal de los talentos y la creatividad de los socios activos en los órdenes de cada una de las unidades de red desde las accionales a las proposicionales, según se mire la ORGANIZACIÓN como subsistema perteneciente a un nicho ecológico del gran sistema ecológico denominado Mundo.

La gestión del conocimiento en una OSCL es un Despliegue Dinámico Aventajado (DDA) que guía y facilita el desarrollo y crecimiento de la organización en cuanto a productividad y competitividad, también ella genera escenarios próvidos, ricos y abundantes en recursividad, sinergia y resiliencia. La gestión del conocimiento tiene un funcionamiento inteligente empresarial $y$ garantiza el alcance pleno de lo misional, al igual que visibiliza, estructura y concreta lo visional, todo conducente a tener un aumento en la rentabilidad del negocio. En la gestión del conocimiento hay trabajo en equipo donde se respeta el individualismo y la cooperación, la investigación y desarrollo es fundamental promisoria y esencial. El conocimiento es: fundamental, imprescindible y rentable. Se tiene efectividad en el negocio y también se hace necesario revelar las relaciones causa - efecto implícitas e invisibles para hacerlas consientes por completo. Todo este despliegue se muestra en la Figura 5.

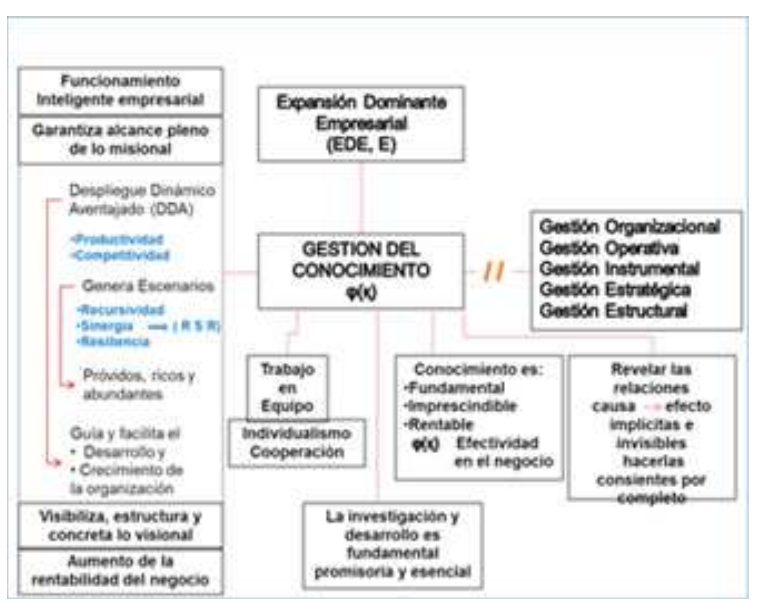

Figura 5. Mentefacto de la Gestión del conocimiento en una OSCL. (Fuente: Propia) 


\section{CONCLUSIONES}

Ante el eminentemente mundo cambiante de hoy día se ha dinamizado el cambio por el aumento del caudal de datos e información disponible y por lo tanto las emergencias y contingencias positivas y negativas que de ellos se derivan reclaman por mayor sensibilidad empresarial para mantener, recuperar o imponer nuevos acoples entre las diferentes conFiguraciones de la realidad empresarial.

Ver la empresa como un sistema abierto en el que se hace gestión del conocimiento que tiene una cultura de organización exitosa permite entender que tanto la resiliencia organizacional como la autopoiesis empresarial son los vórtices de una doble espiral holística que desarrolla: 1. Identidad (marca) y 2. PAE (desarrollo del negocio) como respuesta contundente a la perturbación o turbulencia (P/T). Una organización que impacta de esta forma es una organización sensible coherente y lúcida puesto que: 1 . Detecta y conFigura las fisuras estructurales $\mathrm{y} / \mathrm{o}$ funcionales que se asocian con la P/T interna o externa. 2. Produce cambios micro misionales que reconFiguran el flujo y alcanzan alguno de los diferentes tipos de equilibrio organizacional. 3 . Se avanza a nuevos niveles de riqueza empresarial mediante la creatividad y la innovación.

Queda claro que los sistemas adaptativos complejos son entidades que muestran que se puede alcanzar la estabilidad del sistema apartándose de la linealidad. Ahora bien la propuesta de OSCL asume lo antedicho para los sistemas adaptativos complejos (CAS) como la fase inicial y el desarrollo del sujeto y la empresa como proceso para alcanzar desarrollo social.

Dado que se trata de resolver un problema sistémico, se hace necesario la gestión del conocimiento, lo cual implica apoyarse en sus raíces más profundas y reconocer en los stakeholders tanto en su proyecto de vida como con la organización, para evitar confabulaciones que generen inestabilidad en el sistema; de igual manera se recomienda perseverancia y paciencia para lograr que la organización se convierta en una OSCL.

El funcionamiento inteligente empresarial en una OSCL garantiza alcanzar tanto lo misional como lo visional haciéndolo alcanzable, concreto y sobre todo aumentando la rentabilidad del negocio por encima del tercer cuartil.

\section{Referencias}

[1] Akgun, A., Byrne, J., Lynn, G., \& Keskin, H. (2007). New Product development in turbulent environments: Impact of improvisation and unlearning on new product performance. Journal of Engineering and technology Management, 24, 203-230.

[2] Aldrich, H. (1979). Organizations and environments. Prentice - Hall.

[3] Anderson, C., Paine, F. (1975). Managerial Perceptions and Strategic Behavior. The Academy of Management Journal, 18 (4), 811-823.

[4] Ansoff, I., Eppink, J., \& Gomer, H. (1979). Management of Strategic surprise and discontinuity: problem of managerial decisiveness

[5] Ansoff, Igor [1993]. El planeamiento Estratégico. Nueva tendencia de la Administración. / H Ansoff, R. P. Declerk y Hayes R.I. / Editorial Trillas, México

[6] Barnett, W. \& Carroll, G. (1995). Modeling Internal Organizational Change. Annual Review of Sociology, 21, 217-236.

[7] Becherer, R. \& Maurer, J. (1998). The moderating effect of environmental variables on the entrepreneurial and marketing orientation of entrepreneurled firms, Entrepreneurship Theory and Practice, 22(1): 47-58.

[8] Bennis, W. (1966). Changing organizations: Essays on the development and evolution of human organization. New York: McGraw Hill.

[9] Bourgeois III, L. J.(1985). Strategic Goals, Perceived Uncertainty and Economic Performance In Volatile Environments. Academy of Management Journal, 28(3), 548-573. Retrieved from EBSCO host.

[10] Calantone, R., Garcia, R. \& Droge, C. (2003). The Effects of Environmental 
Turbulence on New product Development Strategy Planning. Journal of product Innovation Management, 20, 90-103.

[11] Collins y Porras. Empresas que perduran. Editorial Norma.

[12] Coutnhbey, H. (2001). 20/20 foresight: crafting strategy in an uncertain world Boston, Mass. Harvard Business School Press.

[13] Dess \& Beard, D. (1984). Dimensions of organizational task environments. Administrative Science Quarterly, 52-73.

[14] Dess, G. G., \& Beard, D. W. (1984). Dimensions of Organizational Task Environments. Administrative Science Quarterly, 29(1), 52-73. Retrieved from EBSCOhost.

[15] Dess, G., Lumpkin, G. \& Covin, J. (1997). Entrepreneurial strategy making and firm performance: tests of contingency and conFigurational models. Strategic Management Journal, vol. 18(9): 677695.

[16] Duncan, R. B. (1972). Characteristics of Organizational Environments and Perceived Environmental Uncertainty. Administrative Science Quarterly, 17(3), 313-327.

[17] Duncan Robert M. (1992), "Quality Forecasting Drives Quality Inventory at GE Silicones," Industrial Engineering, January, pp. 18-21

[18] Emery, F. \& Trist, E. (1965). The Causal Texture of Organizational Environments. Human Relations, 18 (21), 21-32.

[19] Ferber, J (1999) Multi-Agent Systems: An Introduction to Distributed Artificial Intelligence. Addison Wesley

[20] Gray, Ann E., Abrahan Seidmann, and Kathryn-e. Stecke (1993), "A Synthesis of Decision Models for Tool Management in Automated Manufacturing," Management Science, vol. 39, no. 5, May, pp. 549-564.

[21] Georgoff, D.M., and R. G: Murdick (1986), "Manager's Guide to Forecasting," Harvard Business Review, January February, pp. 110-120.

[22] Heisig, P., Vorbeck, J. (2001): Benchmarking Survey Results. In: Mertins, K., Heisig, P., Vorbeck, J. (Eds.):
Knowledge Management. Best Practice in Europe. Berlin, Heidelberg: SpringerVerlag, 1st. Ed., p. 97-123

[23] Kauffman, S. (1993) The Origins of Order: Self Organization and Selection in Evolution. Oxford University Press

[24] Mainzer, K. (1996) Thinking in Complexity: the Complex Dynamics of Matter, Mind and Mankind. Second Edition, Springer

[25] Maruyama, M. (1963) The second cybernetics: Deviation-amplifying mutual causal processes' American Scientist, 51: 164-79

[26] Maturana, H. \& Varela, F. (1992) The Tree of Knowledge. Shambhala

[27] Maturana, H. (1997) Workshop at the Open University, March

[28] Milliken, F. J. (1987). Three Types of Perceived Uncertainty About the Environment: State, Effect, and Response Uncertainty. Academy of Management Review, 12(1), 133-143.

[29] Meza J.J, Bejarano Lilian (2000). Realización de proyectos de vida en las organizaciones sensibles coherentes y lucidas. Revista Ingeniería Universidad Distrital Francisco José de Caldas. 5(1). 82-86

[30] Mitleton, K. E. (2003). Ten principles of complexity \& Enabling Infrastructures. In Complex systems and evolutionary perspectives of organizations: The application of complexity theory to organizations. London: London School Economics.

[31] Probst, G.J.B. (2002): Putting knowledge to work: Case-writing as a knowledge management and organizational learning tool. In: Davenport, Th.; Probst, G.J.B. (Eds.): Knowledge Management Case Book. Best Practices. Erlangen: Publicis and Wiley, 2nd. Edition, p. 312-323

[32] Sallenave, J. P. (2002). La gerencia Integral: No le tema a la competencia, témale a la incompetencia. Ed. Norma.

[33] Serrano González J. Manuel, Carranza Carnicero José Antonio Brito De La Nuez Alfredo Gustavo. Los componentes básicos del equilibrio cognitivo y las 
formas posibles de equilibración cognitiva según la Escuela de Ginebra Anales Psicología N.O 3, 1986 Págs. 109-127

[34] Strategic Goals, Perceived Uncertainty, And Economic Performance in Volatile Environments 1975 "Guidehnes for the empirical classification of organizations."

[35] Volberda, H. W. (1997). Building flexible organizations for fast-moving markets. Long Range Planning, 30 (2), 169-183.

[36] Scott, W. Richard (1981) Organizations: Rational, Natural, and Open Systems. Englewood Cliffs, NJ: Prentice- Hall,
[37] Robert Storey 1973 "On the measurement of the environment: An assessment of the Lawrence and Lorsch environmental uncertainty questionnaire,"Administrative Science Ouarteriy, 18: 27-36.

[38] Tung, Rosalie L. 1979 "Dimensions of organizational environments: An exploratory study of their impact on organization structure," Academy of Management Journal, 22:672-693 